\title{
Un estudio de la dimensión dialógica de pensamiento crítico en estudiantes de medicina de la Universidad Antonio Nariño
}

\section{A Study of the Dialogic Dimension of Critical Thinking in Medical Students at the Antonio Nariño University.}

\author{
Luis Eduardo Olarte", Magally Escobar Martínez", \\ Gustavo Jaimes Monroy
}

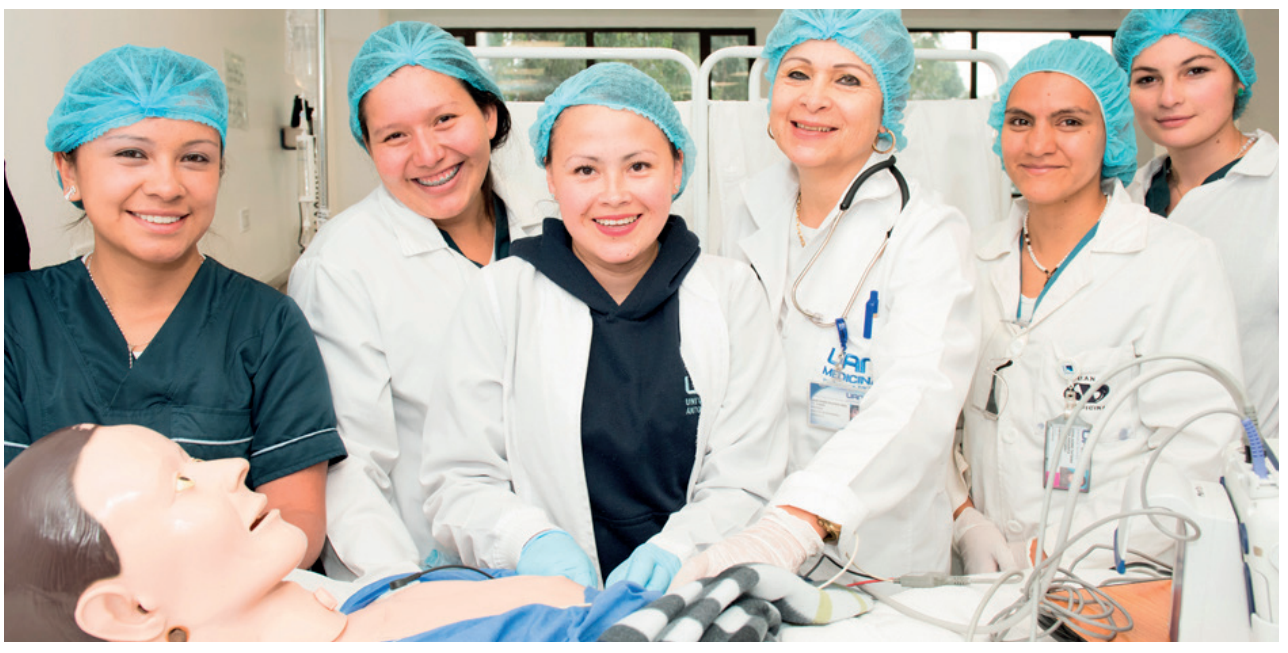

\section{Resumen}

Las investigaciones acerca del pensamiento crítico y los diferentes ámbitos que las componen en la actualidad representan un reto, esto debido a la gran variabilidad de conceptos e interpretaciones de acuerdo con los autores y a los múltiples componentes, enfoques y tipos de estudios que "per se" este permite desarrollar. La gran diversidad de los enfoques investigativos del pensamiento crítico ha permitido que los estudios puedan dirigirse a diversas poblaciones, principalmente en los entornos

Citar este artículo como: Olarte, L. E., Escobar Martínez, M. y Jaimes Monroy, G. (2020).

Un estudio de la dimensión dialógica de pensamiento crítico en estudiantes de medicina de la Universidad Antonio Nariño. Revista Papeles, 12(24), 91-102.

Fecha de recibido: septiembre 5 de 2020. Fecha de aceptado: diciembre 10 de 2020.

* Estudiante de la Facultad de Medicina de la Universidad Antonio Nariño. Correo electrónico: lolarte606@uan. edu.co

** Docente investigadora. Línea de investigación en Educación Médica. Facultad de Medicina. Universidad Antonio Nariño. Correo electrónico: directorudci.medicina@uan.edu.co

*** Docente investigador. Facultad de Odontología. Grupo de Investigación en Salud Oral. Universidad Antonio Nariño. Correo electrónico: tavojaimes@uan.edu.co 
educativos, siendo la mayoría de estos estudios en el contexto de la evaluación de los procesos de aprendizaje, en los que se ven implicados aspectos como las metodologías educativas, la preparación de los docentes frente al pensamiento crítico, las estrategias de enseñanza entre otras, que suponen un conjunto de herramientas necesarias para la promoción y desarrollo del pensamiento crítico en los estudiantes.

Palabras clave: pensamiento crítico, dimensión dialógica, educación.

\begin{abstract}
Research on critical thinking and the different areas that make them up today represent a challenge, due to the great variability of concepts and interpretations according to the authors and the multiple components, approaches and types of studies that "per se" this allows to develop. The great diversity of investigative approaches to critical thinking has allowed studies to target diverse populations, mainly in educational settings, the majority of these studies being in the context of evaluating the learning processes, in which aspects are involved such as educational methodologies, the preparation of teachers on critical thinking, teaching strategies, among others, which represent a set of tools necessary for the promotion and development of critical thinking in students.
\end{abstract}

Keywords: critical thinking, dialogical dimension, education.

\title{
Introducción
}

En la actualidad, el pensamiento crítico es un concepto que abarca un sinnúmero de aspectos, enfoques y perspectivas; esto se debe a que no se ha podido llegar a un consenso, entre los académicos, de lo que supone y compone el pensamiento crítico en sí. Aunque hay asociaciones que permiten establecer una definición del mismo, y lo que lo compone, tal como es el caso de la asociación americana de psicología, y la definición del pensamiento crítico sigue actualmente teniendo una gran variabilidad de conceptos, encontrados en la literatura por los diferentes autores.

Se evidencia que la mayoría de los estudios en pensamiento crítico son enfocados hacia la evaluación del pensamiento social cotidiano sobre problemáticas actuales y acerca de los procesos, metodologías y estrategias implicadas en el aprendizaje en la educación básica y superior (Leontina et al., 2011). Las diferentes investigaciones en el campo de educación superior se han enfocado en evaluación de las creencias, habilidades, roles y experiencias de los profesionales en formación, esto se puede apreciar en estudios como el realizado por Stupple y colaboradores (2017) en el que evidenciaron que al utilizar un kit de herramientas que permitiera el desarrollo del pensamiento crítico, se generaba una prueba de fácil aplicación y que permitía evaluar las actitudes y creencias sobre el pensamiento crítico de los estudiantes de psicología de la universidad de Derby en el Reino Unido. En otros contextos como la investigación de Rosefsky y Saavedra (2017) acerca de si las universidades cultivan el desarrollo del pensamiento Crítico, se evidenció que sí hay un incentivo para el desarrollo del pensamiento crítico en sus diferentes aspectos, pero que esto dependerá mucho de factores como el profesorado, las metodologías y las competencias a adquirir, entre otros factores que representan un alto impacto en el desarrollo de habilidades críticas y reflexivas en los estudiantes durante su formación. Ahora, si se observa desde campos más específicos 
como el área de la salud, se ha visto que los estudios sobre esta temática predominan en estudiantes de pregrado de odontología y con mayor frecuencia en enfermería, enfocándose en las habilidades adquiridas en la práctica y los procesos de aprendizaje durante su formación básica (Leontina et al., 2011). Sin embargo, los estudios dirigidos a los procesos de formación en el campo del pregrado de medicina son escasos tanto en contextos de la evaluación de habilidades adquiridas durante la formación profesional como la adquisición de procesos que motiven el desarrollo del pensamiento crítico, esto permite ver que hay un vacío de los aspectos anteriormente nombrados en esta área de la salud.

\section{Pensamiento crítico}

El pensamiento crítico a lo largo del tiempo ha tenido muchas definiciones y concepciones por distintos autores, así como distintas interpretaciones por las diferentes áreas del saber que se dedican a describirlo y generar nuevo conocimiento. Es por ello, que en busca de una definición base en la que se unificaran los conceptos e interpretaciones, el consenso realizado por la asociación americana de psicología logró definir el pensamiento crítico como "la formación de un juicio autorregulado para un propósito específico, cuyo resultado en términos de interpretación, análisis, evaluación e inferencia pueden explicarse según la evidencia, conceptos, métodos, criterios y contexto que se tomaron en consideración para establecerlo", estableciendo así un concepto unificado que, aunque comprende múltiples perspectivas, permite limitar la variabilidad de las concepciones subjetivas que se le han atribuido (Stupple et al, 2017; Harasym, P.et al., 2008).

\section{El pensamiento crítico en la educación superior}

En la actualidad, la educación superior insiste en la aplicación de procesos educativos que se encuentran apartados muchas veces de la realidad y que los alumnos experimentan en su día a día, promoviendo, en ocasiones, un estilo de aprendizaje memorístico y poco representativo que no solo se encuentra fuera de las motivaciones de los mismos, sino que tampoco
Pensamiento crítico es la formación de un juicio autorregulado para un propósito específico, cuyo resultado en términos de interpretación, análisis, evaluación e inferencia pueden explicarse según la evidencia, conceptos, métodos, criterios y contexto que se tomaron en consideración para establecerlo

cumple con los objetivos de dichos sistemas de aprendizaje; esto no solo produce un aprendizaje superficial sino que repercute en que los estudiantes no integren y apropien el conocimiento previo con el nuevo ni mucho menos contextualizar y/o aplicar adecuadamente el conocimiento. Obteniéndose así, como consecuencia, una dificultad tanto en la capacidad crítica y reflexiva, como en el fomento de la creatividad (Lazo y Herrera, 2011, p.82).

Matthew C. et al. (2018), en su estudio acerca del desarrollo del pensamiento crítico en la educación de profesionales de la salud, menciona que a nivel institucional, parece ser beneficioso el utilizar rutinariamente evaluaciones basadas en la evidencia luego de la apropiación de diferentes conocimientos para medir el desarrollo cognitivo e incluido el pensamiento crítico. Se evidenció que esta 
estrategia parece mejorar de alguna manera el pensamiento crítico de los estudiantes; no obstante,cabe destacar que muchos estudiantes pueden, desde el inicio de su educación superior, ser muy buenos pensadores críticos y, que con la formación superior, crecer a mayores niveles en esta habilidad. Sin embargo, estas son características poco confiables y difíciles de determinar por la variabilidad de las mismas (Reale, Riche, Witt, Baker y Peeters, 2018).

Steffens et al. (2017) en su estudio realizado acerca los niveles de pensamiento crítico en estudiantes de Universidades en Barranquilla describió que las prácticas educativas que hoy en día se desarrollan en los primeros semestres de educación superior de distintas áreas del saber, muy pocas veces llegan al alcance del desarrollo del pensamiento crítico y las competencias necesarias que exige la realidad actual, dificultando de esta manera la configuración de tal pensamiento y su evolución en los futuros profesionales (Steffens, 2017).

Como lo menciona Betancourth et al. (2017), es que en Colombia y en general en las instituciones de educación superior, dentro del plan de formación de las diferentes áreas del saber, tienen contemplada la implementación de metodologías y estrategias que permitan fortalecer y desarrollar el pensamiento crítico y reflexivo durante la formación, representando un aspecto importante y en el que se ven fuertemente implicados los docentes; sin embargo, más allá de lo estipulado en estas estrategias, no se sabe si realmente se aplican y se obtiene el objetivo de estos procesos, ya que muy pocas veces las mismas instituciones que aplican estos procesos educativos, en pro del desarrollo del pensamiento crítico, no se cercioran de evaluar si están dando resultados positivos en los estudiantes; esto puede deberse no solo a la complejidad, quizás a la evaluación de los procesos críticos adquiridos sino también por el poco interés por parte de la institución de obtener este conocimiento (Betancourth et al., 2017 pp-199-223).

\section{El impacto de los docentes y los métodos de enseñanza del pensamiento crítico}

Uno de los aspectos más complejos y discutidos en la literatura es acerca de cómo enseñar o contribuir en el desarrollo del pensamiento crítico en los estudiantes, ya que no se ha podido establecer una estrategia aplicable $y$ con evidencia fuerte para este fin; sin embargo, este aspecto está condicionado a que los educadores deben contar con una preparación para la enseñanza de habilidades del pensamiento crítico previa preparación con la que muchas veces no se cuenta o no se adquiere por diversas causas. No obstante, la adecuada integración y desarrollo del pensamiento crítico no solo depende de las herramientas que se le brindan al individuo para su formación, sino que también supone un proceso en el que se ven implicadas las disposiciones del pensamiento, la motivación, los componentes emocionales al aprender y los procesos específicos para la resolución de problemas (Sufian, 2016).

De acuerdo con lo mencionado, la preparación por parte de los docentes de educación superior para el desarrollo del pensamiento crítico en los estudiantes tiene un gran impacto en la formación de profesionales; sin embargo, la formación de los docentes de las diferentes áreas del saber es diversa y provoca diferentes concepciones y enfoques al implementar y aplicar metodologías para el desarrollo del pensamiento crítico. Esto se puede ver de manera sencilla partiendo de la concepción que tienen sobre el mismo los docentes de las áreas de la salud. En un estudio realizado por Kahlke y Eva (2018) se evidenció que en los docentes de estas áreas se encontraron concepciones del pensamiento crítico con tres enfoques diferentes: biomédico, humanista y de justicia social. Se destacó en este estudio, en el área de la medicina que la concepción biomédica se evidencia como un proceso sistemático de recopilación y análisis de datos, el 


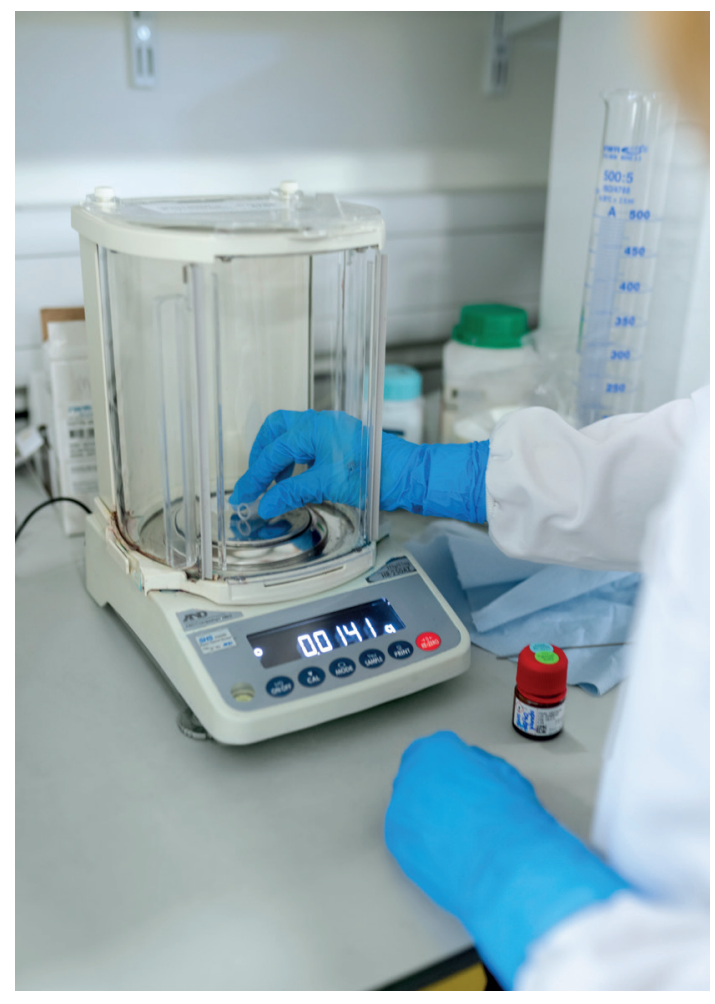

cual se parecía al razonamiento clínico. De esto se puede inferir que de acuerdo a la rama del conocimiento el desarrollo del pensamiento crítico está asociado a un concepto o práctica del área de saber especifico y que también esta percepción no solo se presenta en estos docentes del área de la salud sino también en otras ramas del saber.

Hasta hace unos pocos años los métodos de enseñanza más asociados a la promoción del desarrollo del pensamiento crítico fueron la técnica incorporada o modelo de inmersión, la cual implicaba en la mayoría de los casos una preparación y dominio de estrategias por los educadores acerca de cómo enseñar a los estudiantes desde su disciplina el pensamiento crítico en contexto. Aunque estas estrategias mostraron mejores resultados en los estudiantes de educación superior en diversas áreas del saber, actualmente, se considera que más que estas metodologías, la adecuada formación del pensamiento crítico se constituye en 3 aspectos principales, estos son: la oportunidad de diálogo (estudiantedocente), planteamiento de problemas, ejemplos y tutoría. Estos tres aspectos llevados en conjunto han demostrado una mejor integración y desarrollo del pensamiento crítico en los estudiantes (Smith et at., 2018). Otra perspectiva son aquellos procesos un poco más centrados hacia aspectos definidos. Esto se puede apreciar en la estrategia de la enseñanza orientada a la actividad (AOT) en la que se ven involucrados procesos de apropiación del conocimiento de manera dialógica y colectiva, dirigidos principalmente hacia el análisis y construcción del conocimiento (Carbogim et al., 2019). Dentro de los aspectos de las estrategias de la enseñanza Mathews, SR y Lowe, K (2011) en su artículo sobre los aspectos de la disposición en el aula, mencionan que las preguntas abiertas, los espacios de participación en el aula y el promover el dominio de temas para su discusión, promueven el desarrollo de una adecuada disposición para la integración de los aspectos del pensamiento crítico. Otras de las metodologías que, aunque se sale de lo abordado, ha tenido gran impacto en el desarrollo de los diferentes aspectos del pensamiento crítico, es el aprendizaje basado en problemas en el que se enfoca hacia el desarrollo de cómo abordar y dar solución a una situación problema enfrentándose directamente a esta con herramientas previamente instruidas.

Dentro de este contexto, la toma de decisiones representa para los educadores (y para el estudiante) un desafío, ya que si se parte desde la perspectiva de concepto teórico esta representaría el resultado del pensamiento crítico, dado que su finalidad busca la resolución de problemas; sin embargo, una buena toma de decisiones abarca más que un conocimiento científico adquirido de libros y en el aula, encontrándose implicadas las motivaciones, experiencias, sentimientos y valores que permiten fortalecer los juicios de un individuo hacia su entorno y hacia sí mismo (Mpatisi Moyo et al., 2019). 
Al abordar los procesos de enseñanza del pensamiento crítico en los profesionales de salud se debe resaltar lo comentado por la Federación Mundial para la Educación Médica en el comité de 2002, en donde se determinó que el pensamiento crítico es uno de los aspectos en la formación médica que representa en el profesional de la salud las habilidades para abordar integralmente a los pacientes de manera apropiada y que este forma parte de la calidad del futuro profesional (World Federation for Medical Education, 2002).

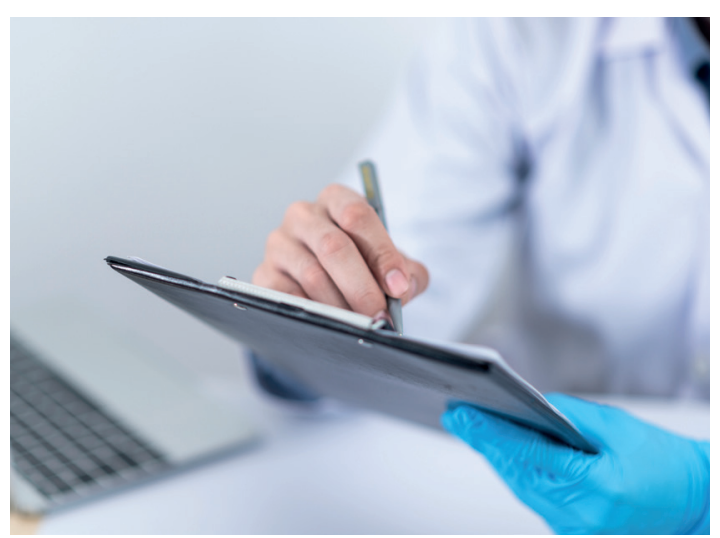

\section{Metodología}

Se realizó una búsqueda en las bases de datos Pubmed, Science Direct, y Google Scholar para el periodo de 2000 a 2018 con el fin de evaluar el pensamiento crítico en instituciones de educación superior enfocadas en áreas de la salud, específicamente enfermería, odontología y medicina. Para la búsqueda se utilizaron las palabras "pensamiento crítico", "pruebas de pensamiento crítico en educación superior", "pensamiento crítico en medicina", "pensamiento crítico en enfermería", "evaluación del pensamiento crítico".

Desde la literatura se seleccionó la prueba de pensamiento crítico, basándose en la información recolectada, seleccionándose una prueba que se enfocara en medir diferentes parámetros de la dimensión dialógica del pensamiento crítico. Para esto se escogió el instrumento de los autores Leontina et al. (2011) el cual, originalmente fue ajustado de los estudios realizados por Santiuste (2001) con el fin de poder evaluar el desarrollo de la argumentación, el debate, el análisis y la convergencia de aspectos del pensamiento crítico en la dimensión dialógica, el cual cuenta con 15 preguntas distribuidas en categorías: expresar y escuchar, dimensión dialógica, expresión escrita y lectura dialógica.

La muestra a conveniencia fue de 40 estudiantes por cada uno de los tres semestres $(n=120)$.
Estos estudiantes cumplían con el criterio de inclusión que consistía en encontrarse tomando todas las asignaturas del semestre correspondiente.

Para adaptar el instrumento se efectuaron tres fases que permitieron obtener un instrumento fiable para la aplicación a la muestra seleccionada.

\section{Adaptación y aplicación de prueba piloto de pensamiento dialógico:}

En la primera etapa de la investigación se llevó acabo la adaptación al lenguaje Colombiano del instrumento aplicado por Leontina et al. (2011), Leontina H (2011) en su estudio del pensamiento crítico y una intervención según disponibilidad de cada uno de los grupos por semestre acerca de los conceptos básicos del pensamiento crítico, las dimensiones del pensamiento crítico y su contexto (explicando en qué consisten y que evalúan); así mismo, se explicaron los objetivos del proyecto de investigación. Posteriormente, se realizó el proceso de inclusión de los participantes mediante el aval del consentimiento informado y se aplicó la prueba piloto a una muestra de 15 estudiantes del instrumento de pensamiento crítico. 


\section{Prueba global y análisis inicial de resultados}

En la segunda etapa de la investigación se aplicó la prueba al total de la población (grupos de estudios de I, IV Y VII semestre de medicina) y al obtenerse sus resultados, se procedió a su análisis por medio de una metodología estadística descriptiva univariada y bivariada para el reconocimiento y asociación entre variables.

\section{Análisis estadístico y conclusiones de resultados}

Una vez obtenidos los resultados y análisis de los mismos, se procedió a su interpretación de manera individualizada y en conjunto para la formación de conclusiones y el reconocimiento del estado de la dimensión dialógica de pensamiento crítico, representándose mediante gráficos estadísticos.

\section{Resultados}

\section{Instrumento de pensamiento crítico}

\section{Gráfico 1. Dimensión diálogica del pensamiento crítico}

\section{Items}

$\mathrm{Al}$ presentar una idea adquirida luego de una búsqueda bibliográfica menciono la fuente(s) de la que proviene.

$\mathrm{Al}$ realizar una búsqueda bibliográfica sé diferenciar entre fuentes que contienen una información confiable y aquellas que no.

Al evaluar un caso clínico tengo en cuenta otras interpretaciones aparte de la realizada por el docente.

Identifico la información relevante de la irrelevante al realizar una búsqueda bibliográfica.

Identifico la idea y contexto principal de cualquier texto

Al escribir sobre un tema médico particular puedo diferenciar entre la evidencia actual y las opiniones de expertos.

Diferencio los hechos y las opiniones de expertos en los textos que leo.

$\mathrm{Al}$ debatir textos propuestos en clase busco interpretaciones alternativas a las que ya han sido mencionadas.

$\mathrm{Al}$ presentar un argumento durante un debate evalúo otros puntos de vista diferentes que sustenten mi interpretación inicial.

En la realización de un trabajo escrito, además de la información principal que lo sustenta, expongo opiniones alternativas de otras fuentes.

Al tener puntos de vista contrapuestos con una bibliografía específica busco información que justifique mis argumentos.

En la realización de un trabajo escrito tengo en cuenta interpretaciones alternativas frente a la idea principal, siempre que sea posible.

Cuando tengo puntos de vista diferentes a los de una bibliografía corroboro la validez de mis argumentos y evalúo la posibilidad de estar equivocado.

$\mathrm{Al}$ adquirir un nuevo conocimiento de una fuente bibliográfica rectifico y pongo a prueba la validez de la información revisando otras fuentes bibliográficas.

Al tener conceptos y puntos de vistas iguales con una bibliografía específica pongo a prueba la validez de la información revisando otras fuentes bibliográficas.

Fuente: propia 
El instrumento aplicado constó de 15 preguntas adaptadas al lenguaje colombiano, con las cuales se evaluaron aspectos de dimensión dialógica, habilidades de escucha y argumentación, expresión e intepretación escrita y lectura dialógica, midiendo las respuestas mediante una escala likert de 5 parámetros (siempre/ casi siempre/ algunas veces/muy pocas veces/nunca). Durante su aplicación el instrumento no tuvo límite de tiempo para su realización.

Respecto a los datos sociodemográficos, se tuvo una proporción de individuos similar a la procedencia, evidenciándose que un $55 \%$ de los encuentados son propios de la capital del país y el otro $45 \%$ proceden de pueblos y ciudades cercanas.

En la evaluación de las métricas utilizadas para la evaluación de la prueba aplicada se encontró que la confiabilidad de la escala tipo likert en la encuesta acerca del estado del pensamiento crítico en su dimension dialógica fue buena. Esto determinado por el valor del alfa de cronbach obtenido (0.883), lo que permite inferir que los ítems poseen una buena redacción y correlación con la variable de respuesta.

\section{Análisis, resultados globales, estadísticas y correlaciones del estudio}

Para el grupo de los estudiantes de primer semestre de medicina se hizo evidente que en general las respuestas estuvieron entre "casi siempre" y "algunas veces", con 174 y 222 respuestas correspondientemente; de lo que se infiere que en este grupo de estudio la mayoría delos estudiantes implementan en su formación diaria muchos de los aspectos contemplados en la dimensión dialógica del pensamiento crítico; sin embargo, se encontró que en la pregunta acerca de la " exposición de ideas alternativas en trabajos escritos" (pregunta número 10) y "al escribir sobre un tema del área de la salud..." (pregunta número 6) tuvo el mayor número de respuestas "algunas veces" (13 y 18 respuestas correspondientemente). Este resultado puede ser percibido como una oportunidad de mejora y refuerzo en los estudiantes en la forma de abordar artículos y la compresión de lectura de los mismos.

Gráfico 2. Items de menor fortaleza en grupo de primer semestre

Pregunta 6. Al escribir sobre un tema médico particular puedo diferenciar entre la evidencia actual y las opiniones de expertos.

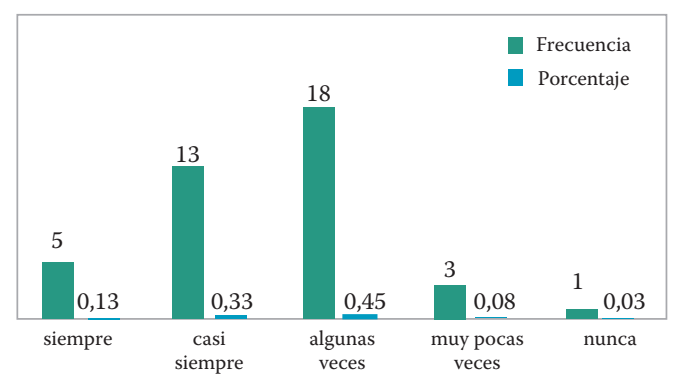

Fuente: propia
Pregunta 10. En la realización de un trabajo escrito además de la información principal que lo sustenta expongo opiniones alternativas de otras fuentes.

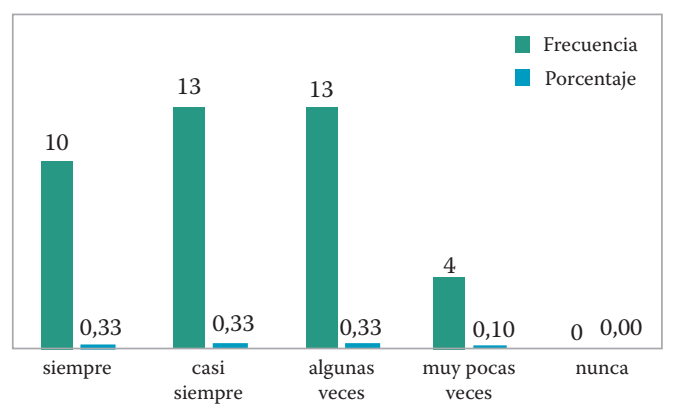

Fuente: propia 


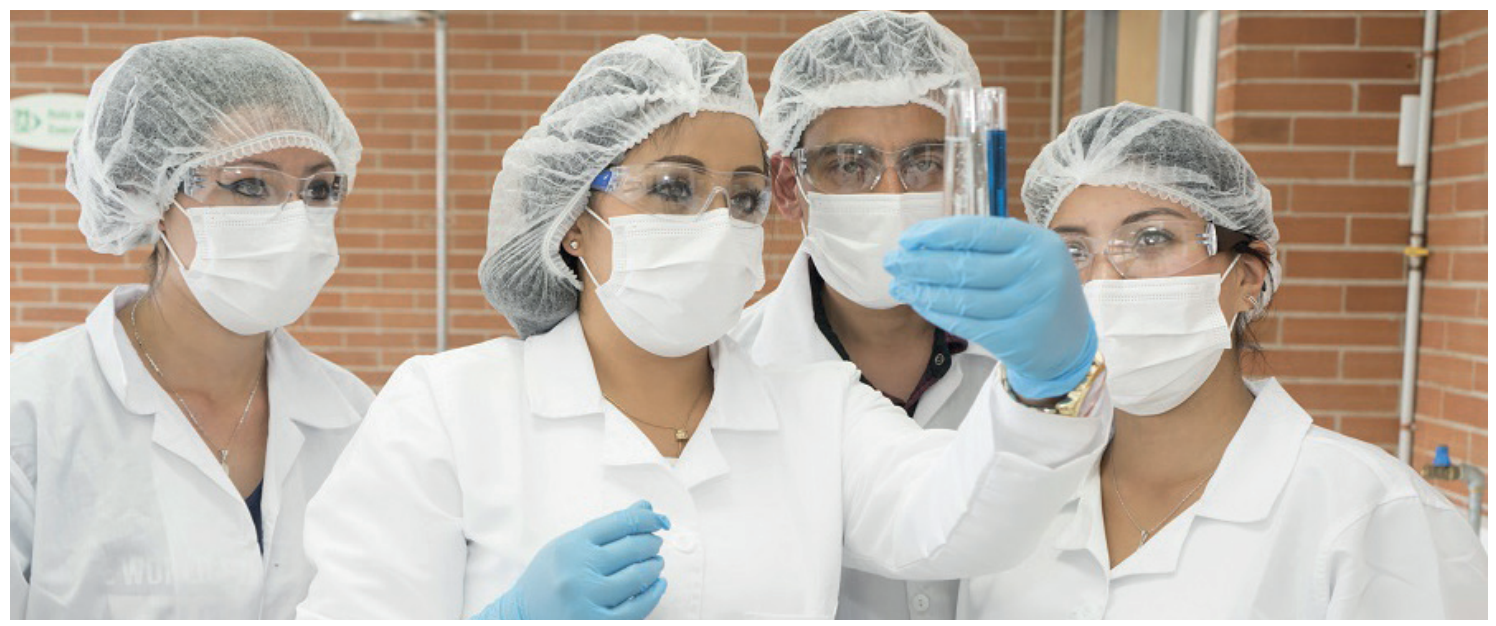

En cuanto a los estudiantes de cuarto semestre de medicina, se evidenció que en general la respuesta en mayor proporción para cada pregunta fue "casi siempre" (total respuestas 264/ promedio porcentual 44\%) permitiendo inferir que en los procesos interrogados sobre la dimensión dialógica del pensamiento crítico puede que se encuentren mejor integrados en esta etapa de formación académica y aunque no se podría saber con exactitud el por qué de este predominio en las respuestas, sí se podría atribuir a los procesos y el avance que se ha obtenido durante la formación académica. Se encontró también que la pregunta con mayor variabilidad en sus respuesta fue acerca de la autoevaluación de la validez de los conceptos adquiridos por un individuo.

Para el grupo de los estudiantes de octavo semestre de medicina se obtuvo una frecuencia de respuestas similar a la obtenida por el grupo de primer semestre, a excepción de las preguntas asociadas a busqueda bibliográfica, identificación de información relevante y reevaluación del conocimiento adquirido, se evindenció en estos items que el grupo de estudiantes mostraron un dominio y/o fortaleza en estos componentes de la dimensión dialógica. Sin embargo, mostraron en el item acerca de la justificación de argumentos propuestos por el individuo un valor significativo de respuestas "muy pocas veces" (correspondiente al 25\% de los individuos del grupo de estudio), esto puede describir una oportunidad de mejoramiento en los sujetos evaluados.

Al realizar el análisis global de las respuesta del total de la población se encontró que los items en relación a la búsqueda de bibliografía y la interpretación de la misma representaron la mejor frecuencia de respuestas "siempre" y "casi siempre" permitiendo inferir que la mayoría de los estudiantes presentan una fortaleza en cuanto a estos aspectos de la dimensión dialógica, en contraposición y aclarando que aunque no hubo alguna pregunta en la que predominaran totalmente respuestas "algunas veces", "muy pocas veces" o "nunca" sí se obtuvo una alta frecuencia (número de respuestas correpondientemente: $40 / 12 / 1$ ) de estas respuestas en el item enfocado en la evaluación de la formación de argumentos e interpretaciones para el debate del conocimiento adquirido. Esto puede demostrar que la adquisición de esta hablidad en los estudiantes representa un reto para la adecuada integración y desarrollo del pensamiento dialógico, a pesar de tener fortalezas en la construcción del conocimiento, evidenciando como anteriormente se ha nombrado, una oportunidad de mejoramiento a partir de nuevas estrategias y/o metodologías innovadoras que permitan el desarrollo de bases de argumentación y juicios en el estudiante en cualquier contexto determinado. 
Gráfico 3. Análisis estadístico de item, argumentación y debate.

Pregunta 9. Al presentar un argumento durante un debate evalúo otros puntos de vista diferentes que sustenten mi interpretación inicial (grupos primer semestre / cuarto semestre y octavo semestre).
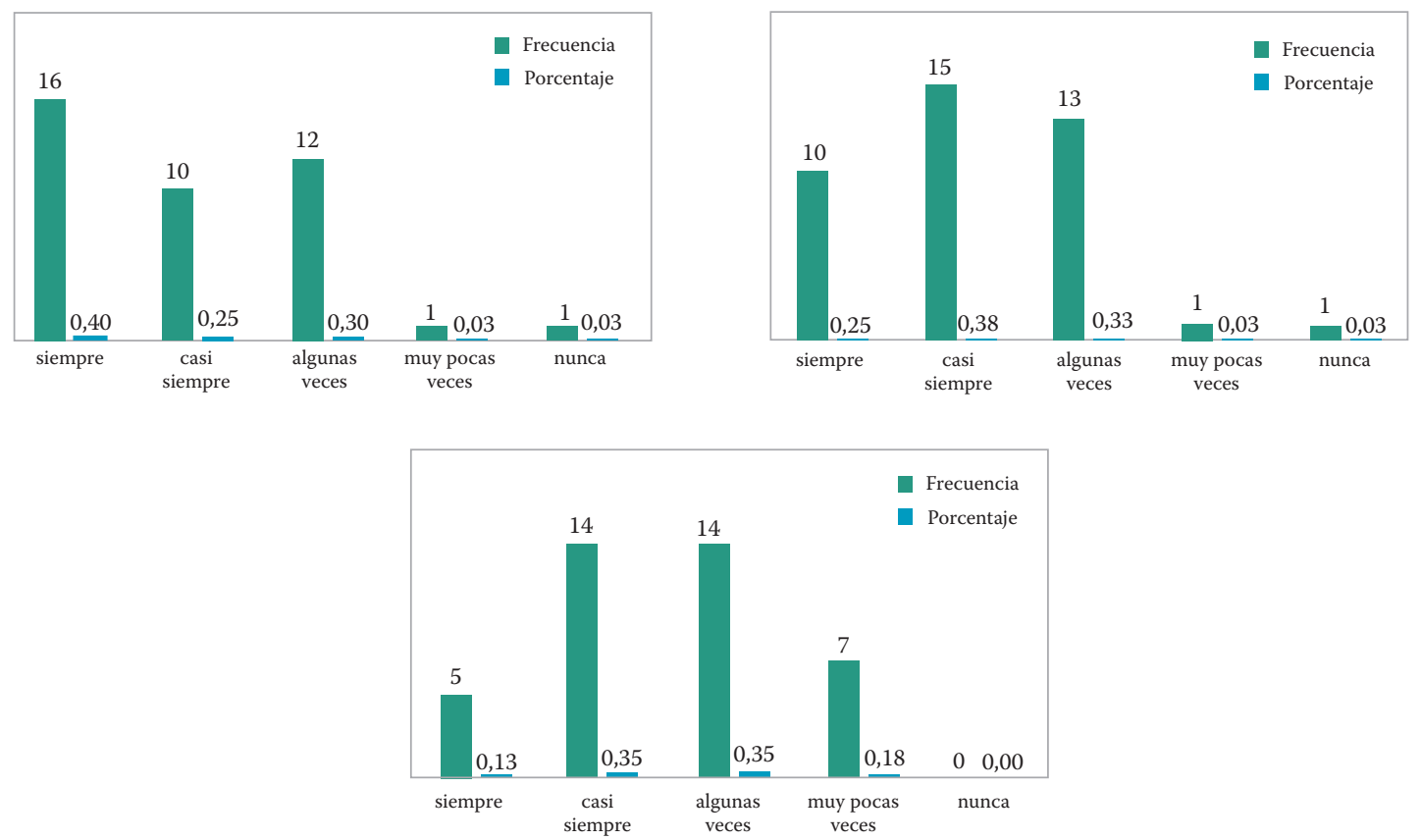

Fuente: propia

En la aplicación de correlaciones de pearson en el estudio se evidenció que los ítems tenían buena redacción (indice de correlación superior a 0.5 para cada una de las preguntas) y estaban correlacionados con las variables de respuesta. Se evidenció que las correlaciones fueron positivas y significativas para las 15 preguntas, indicando que todas las preguntas adaptadas fueron adecuadas para la finalidad del estudio.
Para analizar la relación de dependencia y/o independencia de los resultados obtenidos mediante la prueba de chi cuadrado entre las variables de "pensamiento crítico en la dimensión dialógica y "el semestre actual", se obtuvo que la relación de dependencia estaba presente en los aspectos sobre el debate de ideas $\left(\mathrm{Chi}^{2}: 0.16\right)$ y las interpretaciones de ideas alternativas $\left(\mathrm{Chi}^{2}\right.$ : 0.12$)$, mientras que en los otros items evaluados del pensamiento crítico, fueron signficativamente independientes del semestre cursado.

\section{Conclusiones}

De los aspectos evaluados de la dimensión dialógica del pensamiento críticó se evidenció el desempeño de las habilidades tanto de manera individual (por semestres) como en la globalidad de la población, clasficándose de acuerdo a los resultados obtenidos como fortalezas y oportunidades de mejoramiento. De estas, se destacan las habilidades acerca de búsqueda, lectura y compresión de literatura científica, ya que desmostraron índices de respuesta satisfactorios, representando una fortaleza en los estudiantes de todos los semestres 
evaluados. En contraposición, aquellas relacionadas con la integración y apropiación de la información científica que permite el debate de ideas y confrontación de conocimientos presentan en los estudiantes una oportunidad de mejoramiento.

En la construcción de la dimensión dialógica del pensamiento crítico por los estudiantes se evidenció una dependencia en habilidades de escritura científica y reevaluación de los conocimientos propios con el avance de la etapa académica, permitiendo inferir que los procesos académicos promueven y permiten un desarrollo de esta dimensión del pensamiento crítico.

Cabe destacar que puede haber un sesgo relativo en la investigación, ya que al tomar grupos de estudio en diferentes momentos de su formación académica los procesos de formación a los que estuvieron expuestos pueden haber sido diferentes, de lo que se podría suponer que no todos los individuos presentaban las mismas habilidades $y$ desarrollo del pensamiento crítico; esto abre la posibilidad a que los futuros estudios de pensamiento crítico en estudiantes de pregrado de medicina y en general en estudiantes de educación superior sean de manera más detallada acerca del desarrollo del pensamiento crítico: en sus diferentes dimensiones, siguiendo la evolución a través del tiempo de un único grupo de estudio para disminuir el sesgo y generar un conocimiento replicable en otros contextos.

El desarrollo y evaluación del estado de las diferentes dimensiones del pensamiento critico en los estudiantes de educación superior supone un desafió ya que este está influenciado por un sinnúmero de factores; sin embargo, a partir de los procesos educativos superiores, se puede apreciar que su contrucción tiene un avance positivo y que aunque puede ser variable de indivivuo a individuo se evidencia en cada uno de ellos un progreso significativo a través del tiempo.

\section{Referencias}

Betancourth S, Muñoz K, Rosas T. (2017). Evaluation of critical thinking skills in higher education students in the region of Atacama Chile, Prospectiva. Revista de Trabajo Social e intervención social No. 23, enero-junio 2017: pp. 199-223.

Carbogim, F. C., Oliveira, L. B., Toledo, M. M., Diaz, F. B. B. S., Bittencourt, G. K. G. D., \& Püschel, V. A. A. (2019). Active teaching model to promote critical thinking. Revista Brasileira de Enfermagem, 72(1), 293-298. https://doi.org/10.1590/0034-7167-2018-0002

Forawi, S. A. (2016). Standard-based science education and critical thinking. Thinking Skills and Creativity, 20, 52-62. https://doi. org/10.1016/j.tsc.2016.02.005

Harasym, P. H., Tsai, T., \& Hemmati, P. (2008). Current Trends in Developing Medical
Students' Critical Thinking Abilities. The Kaohsiung Journal of Medical Teacher. World Federation for Medical Education. https://doi.org/10.1016/s1607-551x(08) 70131-1

Kahlke, R. y Eva, K. Perspect Med Educ (2018) 7: 156. https:// ezproxy. uan.edu.co :2112/10.1007/s40037-018-0415-z

Leontina H. (2011). Aplicación de un modelo de intervención pedagógica que desarrolla estrategias de pensamiento crítico para estudiantes de carreras del área de las ciencias. Revista electrónica diálogos educativos,11, 81-94. Omitir de aquí en adelante Vol.11, pp. 81-94, Chile.

Magno, C. Metacognition Learning (2010) 5: 137. https://ezproxy. uan.edu.co:2112/ 10.1007/s11409-010-9054-4 
Mathews, SR y Lowe, K. Learning Environ Res (2011) 14: 59. https://ezproxy.uan.edu. co:2112/10.1007/s10984-011-9082-2

Moyo Mpatisi, Shulruf Boaz, Weller Jennifer, Goodyear-Smith Felicity (2019) Effect of medical students' values on their clinical decision-making. Journal of Primary Health Care, 11, 64-74.

Ojeda E S, Martínez, O et al. (2017) Levels of critical thinking in University students in Barranquilla (Colombia), REV ESPACIOS, Volume 38. Barranquilla.

Pu, D., Ni, J., Song, D. y col. BMC Med Educ (2019) 19: 1. https://ezproxy.uan .edu. co:2112/10.1186/s12909- 018-1418-5

Santiuste, V. (coord.), Ayala, C., Barrigüete, C., García, E., González, J., Rossignoli, J., y Toledo, E. (2001). El pensamiento crítico en la práctica educativa. Fugaz Ediciones.

Reale M, Riche D, Witt B et al. (2018). Development of critical thinking in health professions education: A meta-analysis of longitudinal studies. Currents in Pharmacy Teaching and Learning, Elsevier, 10, 7, Julio, pp. 826-833. https://doi.org/10.1016/j. cptl.2018.04.019
Rosefsky, A, Saavedra J.E. (2011). Do colleges cultivate critical thinking, problem solving, writing and interpersonal skills? Economics of Education Review, Elsevier, 30, 1516-1526.

Steffens, E, Ojeda J, Martinez O. (2017). Levels of critical thinking in University students in Barranquilla (Colombia). Revista Espacios, 38(30), 5.

Smith, T. E., Rama, P. S., y Helms, J. R. (2018). Teaching critical thinking in a GE class: $A$ flipped model. Thinking Skills and Creativity, 28, 73-83. https://doi.org/10.1016/j.tsc.20 18.02.010

Stupplea E, Maratos F, Elandera J et al. (2017). Development of the Critical Thinking Toolkit (CriTT): A measure of student attitudes and beliefs about critical thinking. Thinking Skills and Creativity, Elsevier, 23, 91-100.

Takata, Y., Stein, GH, Endo, K. et al. BMC Med Educ (2013) 13: 156. https://ezproxy.uan. edu.co:2112/10.1186/1472-6920-13-156

World Federation for Medical Education. (2002). Global minimum essential requirements in medical education. Medical Teacher, 24(2), 130-135. https://doi. org/10.1080/01421590220120731 